\title{
Michael Embach, Martina Wallner, Conspectus der Handschriften Hildegards von Bingen
}

\section{Laurence Moulinier-Brogi}

\section{OpenEdition}

\section{Journals}

Édition électronique

URL : http://journals.openedition.org/ifha/8799

DOI : $10.4000 /$ ifha. 8799

ISSN : 2198-8943

\section{Éditeur}

IFRA - Institut franco-allemand (sciences historiques et sociales)

\section{Référence électronique}

Laurence Moulinier-Brogi, « Michael Embach, Martina Wallner, Conspectus der Handschriften Hildegards von Bingen », Revue de l'IFHA [En ligne], Date de recension, mis en ligne le 02 novembre 2017, consulté le 24 septembre 2020. URL : http://journals.openedition.org/ifha/8799 ; DOI : https://doi.org/10.4000/ ifha.8799

Ce document a été généré automatiquement le 24 septembre 2020.

(C)IFHA 


\section{Michael Embach, Martina Wallner, Conspectus der Handschriften Hildegards von Bingen}

Laurence Moulinier-Brogi

\section{RÉFÉRENCE}

Michael Embach, Martina Wallner, Conspectus der Handschriften Hildegards von Bingen, Münster: Aschendorff Verlag, 2013, VII + 336 p., $69 €$ 
Michael Embach, qui dirige actuellement la Stadtbibliothek et le Staatsarchiv de Trèves, s'est signalé depuis plus d'une dizaine d'années comme le grand spécialiste de la réception des œuvres de Hildegarde de Bingen (1098-1179) au Moyen Âge et à l'époque moderne. Depuis son habilitation intitulée Die Schriften Hildegards von Bingen. Studien zu ihrer Überlieferung und Rezeption im Mittelalter und in der frühen Neuzeit (Berlin, Akademie Verlag), il n'a cessé de contribuer à une meilleure connaissance du vaste monde que constituent les écrits de la célèbre nonne rhénane, découvrant notamment un témoin fragmentaire inédit de ses Solutiones triginta octo quaestionum dans la Stadtbibliothek de Trèves.

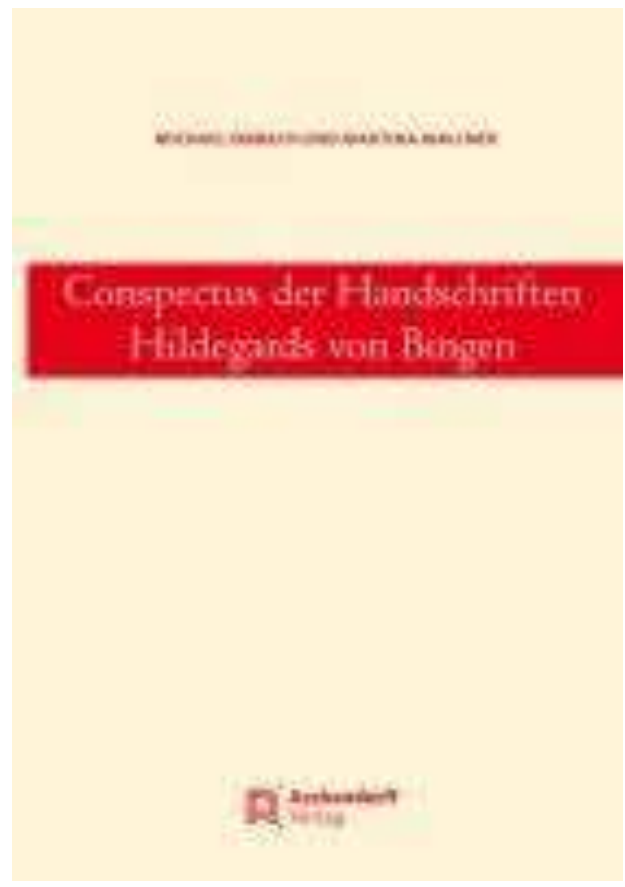

Avec Martina Wallner, il publie le présent Conspectus der Handschriften Hildegards von Bingen, fruit de nombreuses années de travail qui passe en revue l'une après l'autre les bibliothèques du monde entier où sont (ou étaient) conservés, au Moyen Âge ou au début des Temps modernes, des manuscrits des écrits hildegardiens. 363 manuscrits sont ainsi énumérés et décrits, et ce catalogue offre assurément de très solides bases à tous ceux qui veulent se lancer dans ou poursuivre des recherches sur l'œuvre de la visionnaire sans être induits en erreur par la tradition imprimée, les discordances entre manuscrits et éditions atteignant leur climax avec son œuvre médicale (voir à ce sujet le livre tiré de ma thèse, Le Manuscrit perdu à Strasbourg. Enquête sur l'œuvre scientifique de Hildegarde, 1995).

À cette entreprise, dont on ne peut que saluer l'importance et l'utilité, on se permettra d'ajouter deux petits correctifs. Dans la courte préface à ce catalogue, les auteurs expliquent qu'ils ont pris en compte l'œuvre du moine cistercien Gebeno d'Eberbach, le Speculum futurorum temporum alias Pentachronon composé à partir des prophéties de la religieuse, et l'on ne peut qu'approuver ce choix car il n'est pas toujours facile, dans les manuscrits subsistants qui comportent telle ou telle prophétie attribuée à la nonne, de faire le départ entre une prophétie originellement hildegardienne et sa réélaboration par le moine zélé. Les auteurs auraient pu en revanche expliquer au lecteur pourquoi des œuvres comme la Vita domnae Juttae (une hagiographie de Jutta von Spanheim, morte en 1136 et première magistra de Hildegarde), la Vita Sanctae Hildegardis, les Acta Inquisitionis ou encore les Octo lectiones ont été retenues. Ces trois derniers titres en particulier, composés dans des optiques diverses pour perpétuer la mémoire de la sainte (récit hagiographique, actes de l'enquête menée en 1233 en vue d'une canonisation qui n'aboutit d'ailleurs pas au Moyen Âge, et vie abrégée de Hildegarde en prose rimée, pour une lecture liturgique le jour de sa fête, le 17 septembre), sont bien des écrits sur Hildegarde, mais n'émanent nécessairement pas d'elle.

La mention de ces Lectiones nous amène au second point que l'on voudrait rapidement évoquer ici. L'exhaustivité est certes un but quasiment inatteignable, mais on s'étonne 
que ne soit cité qu'un témoin de ce texte, certes le plus ancien (actuellement conservé à Bruxelles) et originaire de Gembloux, où la fête de Hildegarde fut instituée au XIII siècle, alors que selon Monica Klaes, éditrice de la Vita sanctae Hildegardis, on connaitrait aujourd'hui huit témoins des Octo lectiones, le plus souvent transmises avec la correspondance de Guibert de Gembloux, dernier secrétaire de la sainte.

Des remarques similaires peuvent s'appliquer à des textes que l'auteure de ces lignes connaît un peu, par exemple la Lingua ignota, la «langue inconnue » composée par Hildegarde et sur la finalité de laquelle on s'interroge encore (voir récemment l'ouvrage de Sarah L. Higley, Hildegard of Bingen's Unknown Language, 2007). Si l'on en croit l'index final qui permet d'aller directement aux pages décrivant les témoins de telle ou telle œuvre, on ne connaitrait que deux manuscrits de cet écrit. Or cette Lingua ignota figure dument dans le ms. Wiesbaden, Hessische Landesbibliothek, 2, manuscrit géant contenant le canon des œuvres de la sainte et connu comme "Riesenkodex», que M. Embach et M. Wallner ne manquent pourtant pas de décrire (p. 308).

Non contente d'avoir laissé une «langue inconnue ", Hildegarde composa aussi des " litterae ignotae ", un mystérieux alphabet de 23 caractères, conservé dans les codices de Vienne et de Berlin de même que dans le Riesenkodex, qui n'est pas indexé non plus à leur sujet. Mais à ce corpus réduit, on pouvait ajouter d'autres témoins : le ms. Vienne, O. N. B., 1016 ( $2^{\mathrm{e}}$ moitié du XII ${ }^{\mathrm{e}}$ s. et $\mathrm{XIII}^{\mathrm{e}} \mathrm{s}$.), qui contient l'un des livres visionnaires de Hildegarde, le Liber vitae meritorum, et porte la trace de cet alphabet : les fols 116r-121 contiennent des lettres et un chant de Hildegarde avec sa mélodie. On trouve aussi trace de cet alphabet fol.119r, inscrit dans, ou plus précisément sous les lignes de portée. Quant au ms. Stuttgart, Württembergische Landesbibliothek, Cod. Theol. Phil. $4^{\circ}$ 253, appelé " codex de Zwiefalten», s'il est décrit par nos auteurs (p. 249), ils n'indiquent pas dans leur notice qu'il renferme les deux seuls cas connus à ce jour d'une application pratique de cet alphabet et de cette «langue "; l'index final ne permet donc pas d'y parvenir.

Il ne s'agit là bien sûr que de vétilles eu égard à l'énormité et à la valeur scientifique du travail accompli, dans lequel défilent un tel nombre de noms, d'auteurs, mais aussi de copistes et de possesseurs, qu'on se prend à rêver d'un index des noms propres, qui aurait permis de prendre synoptiquement la mesure de la variété des utilisateurs des œuvres de Hildegarde mais aussi des contextes codicologiques dans lesquels elles ont circulé. Mais cela aurait impliqué un surcroit de travail, et une nette augmentation, en volume, d'un livre, on l'aura compris, déjà fort riche! Raison de plus pour l'utiliser sans oublier de se reporter à la somme de Michael Embach, Die Schriften Hildegards von Bingen.

\section{INDEX}

Index chronologique : Moyen Âge

Thèmes : Sources 
AUTEURS

LAURENCE MOULINIER-BROGI

Université Lumière Lyon 2 